\title{
Película biodegradable de almidón de maíz termoplástico y quitosano con actividad antimicrobiana empleada como envase activo
}

\author{
Biodegradable films based on thermoplastic \\ starch and chitosan with antimicrobial \\ activity employed as active packaging
}

\author{
Olivia Valeria López ${ }^{1}$, Luciana Andrea Castillo ${ }^{1,2}$, Sonia Farenzena ${ }^{3}$, \\ Esteban Pintos ${ }^{1}$, María Susana Rodríguez ${ }^{3}$, María Alejandra García ${ }^{4}$, \\ Marcelo Armando Villar ${ }^{1,2}$
}

\author{
${ }^{1}$ Planta Piloto de Ingeniería Química, PLAPIQUI (UNS-CONICET), Camino “'La Carrindanga”' Km 7, Bahía Blanca, \\ Buenos Aires, Argentina. \\ ${ }^{2}$ Departamento de Ingeniería Química, Universidad Nacional del Sur, CP: 8000, Av. Alem 1253, Bahía Blanca, Buenos \\ Aires, Argen-tina. \\ ${ }^{3}$ Instituto de Química del Sur, INQUISUR (UNS-CONICET), Laboratorio de Investigaciones Básicas y Aplicadas en \\ Quitina, LIBAQ-Departamento de Química, UNS, Av. Alem 1253, Bahía Blanca, Buenos Aires, Argentina. \\ ${ }^{4}$ Centro de Investigación y Desarrollo en Criotecnología de Alimentos, CIDCA (UNLP-CONICET- CICPBA), 47 y 115 , \\ La Plata, Buenos Aires, Argentina. \\ e-mail: olivialopez@plapiqui.edu.ar
}

\section{RESUMEN}

Una de las líneas para el desarrollo de envases activos contempla la incorporación de agentes antimicrobianos como el quitosano, polisacárido derivado del exoesqueleto de crustáceos. No obstante, su elevada masa molar restringe su difusión desde el envase hacia el alimento por lo que el empleo de oligómeros de características diferenciales es una alternativa interesante, aún poco investigada. El objetivo de este trabajo fue obtener películas activas a base de almidón de maíz termoplástico (TPS) mediante la incorporación de oligoquitosanos $\left(\mathrm{OQ}\right.$, masa molar $=290 \mathrm{~g} \mathrm{~mol}^{-1}$ y grado de polimerización =2). Estos materiales fueron obtenidos mediante termo-compresión en una prensa hidráulica. Se realizaron ensayos de difusión de los OQ desde la matriz de TPS hacia un medio líquido con diferentes grados de acidez $(\mathrm{pH}=3,5$ y 7). Las condiciones del medio líquido fueron seleccionadas según la acidez de una amplia variedad de alimentos. A partir de estos ensayos, se demostró la capacidad de difusión de los oligómeros y su dependencia con el pH del medio. La eficiencia de la incorporación de los OQ en el material de envase se comparó con la correspondiente al método convencional de spray del alimento con una solución del agente activo. La capacidad antimicrobiana de las películas y de las soluciones de OQ se evaluó a partir del recuento de hongos y levaduras, empleando frutillas frescas como alimento de estudio. Los resultados derivados de estos ensayos evidenciaron el carácter activo de la película desarrollada, demostrándose además la mayor eficacia del uso de envases activos antimicrobianos, respecto de los métodos convencionales.

Palabras clave: película activa - almidón termoplástico - oligómeros de quitosano - difusión - capacidad antimicrobiana.

\section{ABSTRACT}

One of the research lines related to active packaging takes into account the incorporation of antimicrobial agents such as chitosan, a polysaccharide derived from the crustaceans' exoskeleton. However, because of chitosan high molar mass, its diffusion from the package to the food surface is restricted, so the use of oligomers is an interesting but less investigated alternative. The purpose of this work was to obtain active films based on thermoplastic corn starch (TPS) by adding oligochitosans (OC, molar mass $=290 \mathrm{~g} \mathrm{~mol}^{-1}$ and degree of polimerization $=2$ ). Films were thermo-compressed in a hydraulic press. Diffusion assays of OC from TPS matrix to liquid medium with different acidity degree $(\mathrm{pH}=3,5$, and 7) were performed. Liquid 
medium conditions were selected according to the acidity of a wide variety of foods. From these assays, oligomers diffusion capacity and the dependence with $\mathrm{pH}$ were demonstrated. The efficiency of OC incorporation in packaging material was compared to the corresponding to the conventional method by food spray with a solution of the active agent. The antimicrobial capacity of films and OC solutions was evaluated from the count of yeasts and molds, using fresh strawberries as food product. Obtained results from these experiments showed the active character of films based on TPS and OC, and a greater efficiency of antimicrobial active packaging compared to the conventional methods.

Keywords: active film - thermoplastic starch - chitosan oligomers - diffusion - antimicrobial capacity.

\section{INTRODUCCIÓN}

En general, los agentes antimicrobianos se aplican a los alimentos para extender la vida útil del producto mediante métodos directos, siendo el spray la tecnología más empleada a escala industrial. El amplio uso de esta técnica se basa en la simplicidad, costo, y versatilidad para una amplia variedad de productos. Sin embargo, la pérdida de eficiencia del poder activo de la solución es una de las principales desventajas de esta forma de aplicación y puede atribuirse a diferentes factores, induciendo al uso de soluciones de relativamente altas concentraciones (aunque dentro de los establecidos por las regulaciones vigentes). Por un lado, la concentración del antimicrobiano podría reducirse debido a reacciones secundarias con algunos componentes del alimento, disminuyendo así la efectividad del método. Por otra parte, el agente podría migrar rápidamente hacia el interior del alimento, dejando desprotegida la superficie del producto en contacto con el medio. En tal sentido, la incorporación de agentes antimicrobianos a matrices poliméricas es una alternativa interesante para desarrollar envases activos. Así, se lograría una liberación controlada del antimicrobiano hacia la superficie del alimento, manteniendo una concentración efectiva superficial durante tiempos más prolongados, permitiendo así reducir las concentraciones del preservador utilizado. El quitosano, polisacárido obtenido a partir de exoesqueletos de crustáceos, ha demostrado ser un efectivo agente antimicrobiano, especialmente frente a hongos y otras bacterias causantes de enfermedades transmitidas por alimentos (ETAs). Sin embargo, la elevada masa molar del mismo dificulta su difusión desde el envase hacia el alimento. Una opción innovadora sería el empleo de oligoquitosano (OQ) cuyas características diferenciales facilitarían su difusión. Por otra parte, el uso de materiales biodegradables de origen natural para el desarrollo de envases resulta interesante desde el punto de vista ecológico. Dentro de este contexto, el almidón es una alternativa prometedora debido a su bajo costo, amplia disponibilidad y funcionalidad. Adicionalmente, este polisacárido puede ser procesado empleando la tecnología desarrollada para los polímeros sintéticos. Si bien el almidón no es un material termoplástico, el procesamiento del mismo bajo elevados esfuerzos de corte y altas temperaturas, en presencia de plastificantes, permite obtener almidón termoplástico (TPS) [1]. La incorporación de oligómeros de quitosano a matrices de TPS permitiría obtener materiales biodegradables con una funcionalidad adicional. Si bien el desarrollo de películas de almidón con la adición de quitosano ha sido ampliamente estudiada [2,3], son escasos los trabajos que evalúan la incorporación de oligómeros de quitosano a matrices de TPS, como así también la difusión de este derivado. Asimismo, aunque son numerosos los estudios relacionados con los envases activos, que incluyen en su formulación compuestos antimicrobianos, todavía no se ha realizado un análisis exhaustivo de sus aplicaciones con fines de prevenir el deterioro fúngico de alimentos [4]. Además, la mayoría de estos estudios concernientes al efecto de los envases activos sobre hongos y levaduras, se refieren al uso de aceites esenciales [5-7].

El objetivo de este trabajo fue desarrollar películas de almidón de maíz termoplástico (TPS) y quitosano de baja masa molar con el propósito de obtener envases activos para productos frutihortícolas frescos. Los oligoquitosanos (OQ) empleados en este estudio fueron caracterizados empleando técnicas de análisis complementarias. Se optimizaron las condiciones operativas para la obtención de la película activa mediante termocompresión. Se realizó un análisis macro y microestructural de los materiales como así también la determinación de sus propiedades ópticas. La liberación de los OQ desde la matriz de TPS fue llevada a cabo empleando un medio líquido simulante a diferentes valores de $\mathrm{pH}$. Además, se estudió comparativamente el uso de envases tipo bolsa a base de esta película activa y el de una solución enriquecida con los oligómeros para extender la vida útil de frutillas frescas.

\section{MATERIALES Y MÉTODOS}

Se empleó almidón de maíz nativo provisto por Misky-Arcor (Tucumán, Argentina) con un contenido de amilosa de 23,9 \pm 0,7 \% [8]. Los oligoquitosanos (OQ) fueron obtenidos por degradación oxidativa del quitosano asistida por radiación de microondas empleando una modificación del método propuesto por SHAO et al. [9]. El quitosano usado como materia prima (masa molar $=468,200 \mathrm{Da}$ ) fue sintetizado a partir de quitina 
obtenida de residuos de exoesqueletos de langostinos (Pleoticus mülleri) según el método propuesto por ZÚÑIGA et al. [10].

La masa molar promedio y el grado de polimerización de los OQ fueron determinados por el método del análisis del grupo terminal [9]. La caracterización morfológica fue realizada por Microscopía Electrónica de Barrido (SEM) usando un microscopio electrónico JEOL JSM-35 CF con detector de electrones secundarios.

La caracterización estructural se llevó a cabo empleando Espectroscopía Infrarroja con Transformada de Fourier (FTIR) utilizando un espectrofotómetro Thermo Nicolet Nexus. La muestra fue preparada moliendo y mezclando OQ con $\mathrm{KBr}$ (Sigma-Aldrich, $99 \%$ ) al $1 \% \mathrm{~m} / \mathrm{m}$, obteniéndose una pastilla transparente. Los espectros se adquirieron a partir de 100 escaneos acumulados a una resolución de $4 \mathrm{~cm}^{-1}$ en un intervalo de $4000-400 \mathrm{~cm}^{-1}$.

Las propiedades térmicas se determinaron por Calorimetría Diferencial de Barrido (DSC) empleando un calorímetro Perkin Elmer Pyris I. Se pesaron aproximadamente $10 \mathrm{mg}$ de $\mathrm{OQ}$ en cápsulas herméticas para evitar la pérdida de agua durante el ensayo. Las muestras fueron calentadas de 30 a $250{ }^{\circ} \mathrm{C}$ a $10^{\circ} \mathrm{C} / \mathrm{min}$, bajo atmósfera de nitrógeno. A partir del termograma, se determinaron la temperatura de inicio y la de fusión así como también la entalpía asociada a dicha transición térmica. La degradación térmica se estudió mediante Análisis Termogravimétrico (TGA) empleando una balanza TA Instrument Discovery Series. Las muestras se calentaron de 30 a $700{ }^{\circ} \mathrm{C}$ a $10^{\circ} \mathrm{C} / \mathrm{min}$, bajo atmósfera de aire. A partir de las curvas de pérdida de masa en función de la temperatura se determinaron la temperatura de inicio y la de máxima degradación.

Para preparar la película TPS-OQ-TPS se disolvieron oligoquitosanos (OQ) en una solución de ácido acético al $0,1 \% \mathrm{v} / \mathrm{v}$, obteniéndose una concentración de $0,3 \mathrm{~g} / \mathrm{mL}$. Paralelamente, se obtuvieron películas de almidón termoplástico (TPS) empleando las mismas proporciones de los componentes y condiciones de procesamiento ya reportadas en un trabajo previo [11]. La solución enriquecida en OQ fue esparcida sobre el film TPS obteniéndose una capa delgada y homogénea del agente antimicrobiano. Luego, otra película de TPS fue colocada sobre la capa de OQ y el sistema bicapa fue procesado mediante termocompresión en una prensa hidráulica a $90{ }^{\circ} \mathrm{C}$ durante 5 minutos. La concentración final de OQ en la película TPS-OQ-TPS fue $0,12 \mathrm{~g} / \mathrm{g}_{\text {TPS }}$.

La microestructura de la película se estudió por SEM con un microscopio electrónico JEOL JSM-35 $\mathrm{CF}$ con detector de electrones secundarios, usando un voltaje de $10 \mathrm{kV}$. Las películas fueron criofracturadas por inmersión en nitrógeno líquido, se montaron sobre tacos de bronce y se recubrieron con una capa de oro.

El color de la película se determinó en un colorímetro Hunterlab UltraScan XE en modo transmitancia, analizando al menos diez posiciones al azar del material en estudio. Los parámetros de cromaticidad $a$ (verde-rojo) y $b$ (azul-amarillo) así como la luminosidad $(L)$ se obtuvieron según la escala Hunter.

La opacidad de la película se determinó a partir de la integración de la curva espectrofotométrica entre 400 y $700 \mathrm{~nm}$. Para la obtención de dicho espectro, las películas fueron cortadas en rectángulos y colocadas en celdas de $1 \mathrm{~cm}$ de camino óptico, empleándose un espectrofotómetro PG Instruments T60 UV/vis.

La difusión de los OQ desde la película TPS-OQ-TPS se evaluó a partir de la inmersión de especímenes previamente pesados en un volumen determinado de simulante. Estos ensayos se realizaron a temperatura ambiente, empleando medios líquidos simulantes con diferentes grados de acidez $(\mathrm{pH}=3,5$ y 7$)$. Se tomaron alícuotas del medio líquido a distintos tiempos (30,60, 120, 150, y $180 \mathrm{~min}$ ), cuantificándose espectrofotométricamente la concentración de oligómeros según el método de la D-glucosamina descripto por SHAO et al. [9]. A tal fin, se utilizó un espectrofotómetro Spectronic 21D y los valores informados corresponden al promedio de, al menos, cinco determinaciones para cada tiempo y $\mathrm{pH}$ ensayados.

Se evaluó la capacidad antimicrobiana de la película TPS-OQ-TPS y de una solución de OQ empleando frutillas frescas como alimento de referencia. Para llevar a cabo este estudio, se seleccionaron frutas con el mismo grado de maduración, como así también de tamaños y peso similares ( 20 g). Además, las muestras no presentaban signos visibles de daños físicos ni de podredumbre. Cabe destacar, que las frutillas estudiadas no fueron sometidas a ningún pre-tratamiento de sanitización con el propósito reducir la carga microbiana inicial. Para evaluar la capacidad antimicrobiana de la película TPS-OQ-TPS, se obtuvieron envases flexibles tipo bolsa mediante termosellado siguiendo el procedimiento descripto por LÓPEZ et al. [12]. Las frutillas fueron introducidas individualmente dentro de cada uno de los envases a base de TPS-OQ-TPS, preparándose tres réplicas de estas muestras que fueron denominadas $O Q_{\text {pelicula }}$. Por otra parte, otro lote de frutillas fueron sometidas a la técnica de spray, empleando un volumen determinado de solución del agente activo $\left(O Q_{s o l}\right)$. Dicha solución fue preparada de manera similar a la solución enriquecida de estos derivados, previamente descripta. Cabe destacar que el contenido de OQ en los envases de TPS-OQ-TPS era el mismo que el correspondiente al volumen de solución de oligómeros utilizado para el spray de los frutos. Paralelamente, se pre- 
pararon tres réplicas de frutos control que fueron denominados $C$. Todas las muestras fueron almacenadas a $25^{\circ} \mathrm{C}$ y $50 \%$ HR durante siete días. Al concluir dicho tiempo de almacenamiento, se procedió a analizar microbiológicamente los frutos ensayadas. Se tomaron porciones de $10 \mathrm{~g}$ de cada una de las frutillas almacenadas y se procesaron en un homogeneizador Stomacher Seward Model 400 con agua peptonada bufferada (pH $=7,2 \pm 0,2$ a $25^{\circ} \mathrm{C}$ ), previamente esterilizada. De esta manera, se obtuvo un homogeneizado del alimento cuya concentración resultó de $1 \% \mathrm{~m} / \mathrm{v}$. Alícuotas de $1 \mathrm{~mL}$ de cada uno de los homogeneizados se sembraron en placas $3 \mathrm{M}^{\mathrm{TM}}$ Petrifilm ${ }^{\mathrm{TM}}$ Count Plates para el recuento de hongos y levaduras. A partir de cada homogeneizado se prepararon tres réplicas, de manera de disponer de nueve recuentos de hongos y levaduras para $O Q_{\text {película }}, O Q_{\text {sol }}$ y $C$. Todas las placas sembradas fueron incubadas a $37^{\circ} \mathrm{C}$ durante siete días. Luego se hizo el recuento del total de hongos y levaduras y los valores informados fueron expresados como unidades formadoras de colonias (UFC) por gramo de alimento.

Para el tratamiento estadístico de los datos experimentales se utilizó un análisis de varianza (ANOVA), realizándose un estudio comparativo de las características de las películas así como también de los resultados obtenidos en los ensayos de difusión y de capacidad antimicrobiana. Además, se empleó el Test LSD (Least Significant Difference) de Fisher para analizar las diferencias con un nivel de significancia de $p=0,05$.

\section{RESULTADOS Y DISCUSIÓN}

El mecanismo de degradación oxidativa del quitosano en presencia de un agente oxidante y asistida por radiación microondas se representa en la Figura 1. Esta vía de síntesis resulta similar para diferentes agentes oxidantes tales como hipoclorito de sodio y peróxido de hidrógeno.

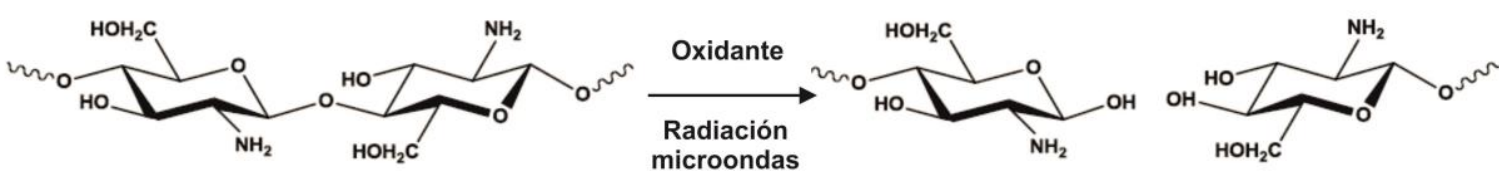

Figura 1: Representación esquemática de la síntesis de oligómeros de quitosano.

La relevancia del estudio de las características estructurales de los OQ radica en que las mismas influyen en la potencialidad de las aplicaciones de este polisacárido de bajo peso molecular. La masa molar promedio de los OQ sintetizados resultó ser de $290 \mathrm{~g} \mathrm{~mol}^{-1}$. Se ha informado que la masa molar de estos derivados depende de las condiciones de reacción. En tal sentido, ZHENG et al. [13] evaluaron el efecto de cuatro variables independientes (relación de quitosano/solución, temperatura, tiempo y cantidad de agente oxidante) sobre la masa molar del derivado sintetizado. Estos autores optimizaron las condiciones operacionales de manera tal de obtener un oligómero de aproximadamente $11000 \mathrm{Da}$. Considerando que la masa molar de las unidades repetitivas de glucosamina es 161, el grado de polimerización de los OQ resultó de aproximadamente 2. Este bajo valor de masa molar supone una mayor movilidad y capacidad de difusión de estos derivados. Esta funcionalidad le otorga un valor agregado a la capacidad antimicrobiana inherente de los derivados de quitosano, ampliando así su campo de aplicación.

En la Figura 2 se muestran micrografías SEM a distintas magnificaciones de los oligómeros de quitosano. Se evidenció la presencia de unidades de forma esférica, con superficies lisas y tamaños uniformes. Morfologías similares fueron reportadas por KHANMOHAMMADI et al. [14]. A partir de las micrografías SEM se pudo determinar el diámetro medio del OQ el que resultó ser de 1,5 \pm 0,2 $\mu \mathrm{m}$, midiendo al menos cien unidades esféricas. 


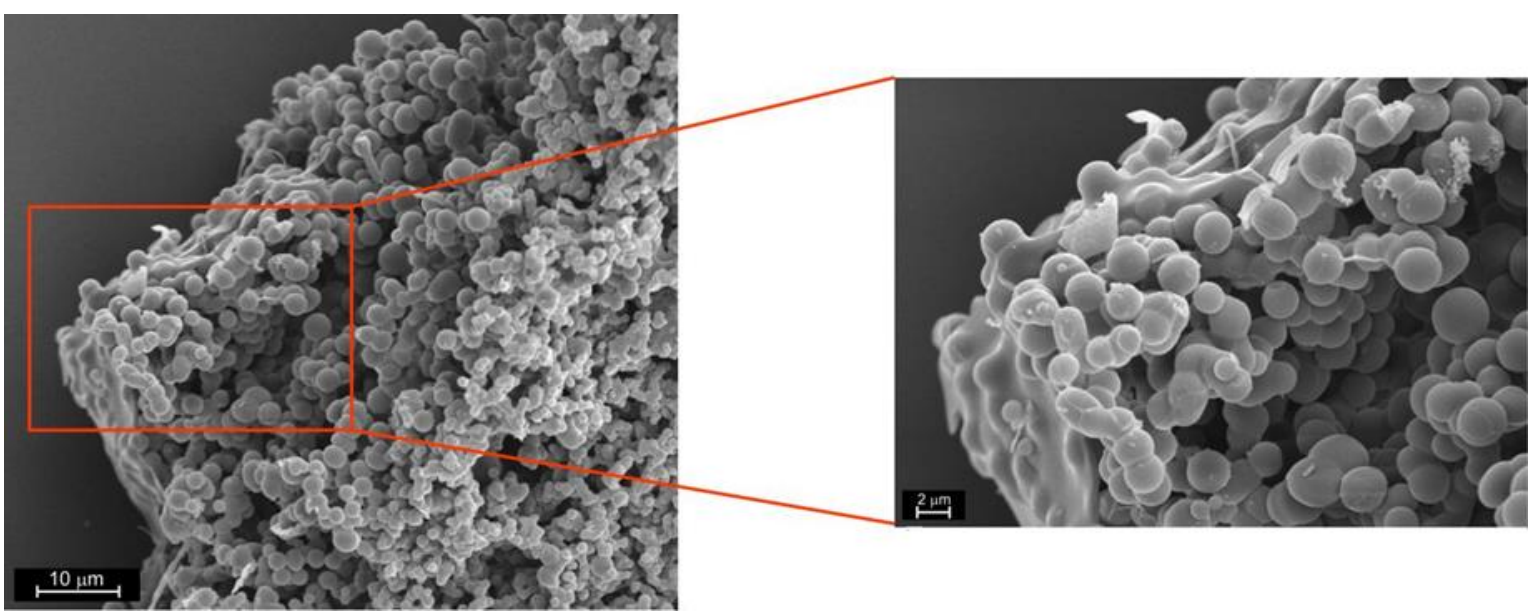

Figura 2: Micrografías SEM de los oligómeros de quitosano sintetizados.

Los espectros FTIR aportaron información relevante para el estudio de la estructura química de este derivado del quitosano. La Tabla 1 presenta la asignación de las principales bandas correspondientes a los grupos funcionales característicos de los OQ. Espectros similares han sido reportados por otros autores [15$18]$.

Tabla 1: Asignación de bandas FTIR de oligómeros de quitosano.

\begin{tabular}{c|c}
\hline NUMERO DE ONDA $\left(\mathbf{c m}^{-1}\right)$ & GRUPO FUNCIONAL \\
\hline 3735 & -OH libres \\
\hline 3450 & $\begin{array}{c}\text { Vibraciones de estiramiento de O-H y N-H } \\
\text { nas alquílicas }\end{array}$ \\
\hline 2918 & $\begin{array}{c}\text { Vibraciones de estiramiento de C-O en los grupos aceti- } \\
\text { los remanentes }\end{array}$ \\
\hline 1653 & $\begin{array}{c}\text { Deformación simétrica de -CH } \\
\text { (1323 }\end{array}$ \\
\hline 1419 & $\begin{array}{c}\text { Vade } \\
1379\end{array}$ \\
\hline 1151 & $\begin{array}{c}\text { Vibraciones de estiramiento de C-OH } \\
895\end{array}$ \\
\hline 1031 & Deformación fuera del plano del -NH \\
\hline 667 &
\end{tabular}

El estudio de las propiedades térmicas de los oligómeros permite determinar las condiciones de procesamiento de estos polisacáridos de bajo peso molecular. Así, se puede establecer el rango de temperatura óptimo que permita la fusión del material, evitando su degradación térmica. La curva de DSC de los OQ evidenció un único evento endotérmico asociado a la fusión de las cadenas poliméricas de estos derivados (Figura 3a). El inicio de esta transición térmica tuvo lugar a los $45^{\circ} \mathrm{C}$, mientras que el pico de fusión se detectó a los $73{ }^{\circ} \mathrm{C}$. Por otra parte, la entalpía asociada a este evento térmico fue de $234 \mathrm{~J} / \mathrm{g}$. El termograma evidenció la presencia de un segundo pico que se atribuye a la fusión del quitosano de partida [19].

La Figura 3b muestra la pérdida de masa de los oligómeros de quitosano sintetizados en función de la temperatura. El análisis del comportamiento de la degradación térmica de los OQ evidenció la ocurrencia de tres etapas de pérdida de peso irreversibles. La primera $(\sim 10 \%)$ está asociada a la evaporación del agua físicamente adsorbida y fuertemente unida por puentes hidrógeno al polisacárido [20]. La segunda etapa corresponde a la degradación de las cadenas poliméricas de los OQ, siendo ésta la más relevante ya que permite determinar las condiciones de procesamiento térmico de estos derivados. Esta pérdida de peso está relaciona- 
da con la despolimerización/descomposición de las cadenas de polímero a través de la desacetilación y del clivaje de las uniones glicosídicas [21]. La degradación térmica asociada a este evento comenzó en $\sim 100{ }^{\circ} \mathrm{C}$, presentando una máxima velocidad de descomposición a $280{ }^{\circ} \mathrm{C}$. Si bien el valor de la temperatura de degradación máxima de los OQ concuerda con el reportado por ZIEGLER-BOROWSKA et al. [22] para quitosano, la temperatura de inicio se desplazó hacia valores significativamente menores. Este resultado puede atribuirse a que el método de degradación oxidativa empleado para la obtención de los OQ alteró la estructura del quitosano, minimizando interacciones intra- e intermoleculares y disminuyendo el peso molecular del polisacárido [13]. El último evento de pérdida de peso corresponde a la destrucción térmica del anillo de piranosa y a la descomposición del residuo carbonoso [20, 23].
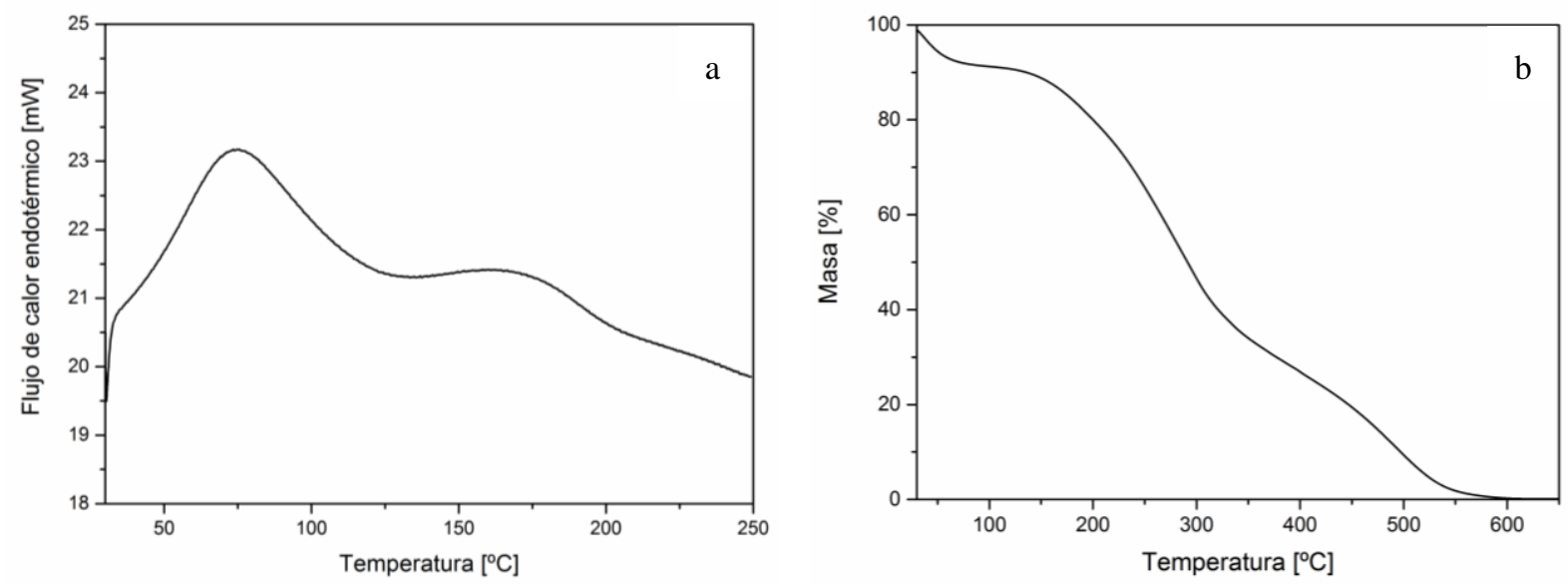

Figura 3: a) Termograma DSC y b) curva TGA de los oligómeros de quitosano sintetizados.

Los oligómeros caracterizados fueron incorporados a una matriz de almidón termoplástico (TPS), obteniéndose la película TPS-OQ-TPS. Macroscópicamente, este material presentó una adecuada flexibilidad y una apariencia homogénea, siendo además fácil de manipular. Por otra parte, resultó ser una película translúcida, con una superficie suave y tonalidad ámbar-amarillenta. A pesar del acabado satinado, la misma no presentó una textura oleosa ni adherente al tacto.

El análisis microestructural de los materiales poliméricos permite estudiar la homogeneidad de su superficie y del área transversal, además de evaluar la compatibilidad entre los diferentes constituyentes. La superficie de fractura de la película TPS-OQ-TPS resultó lisa, sin poros, fisuras, ni canales de migración del glicerol (Figura 4). Esto confirma la evaluación macroscópica de la película respecto de la ausencia de oleosidad o adhesividad al manipular el material.

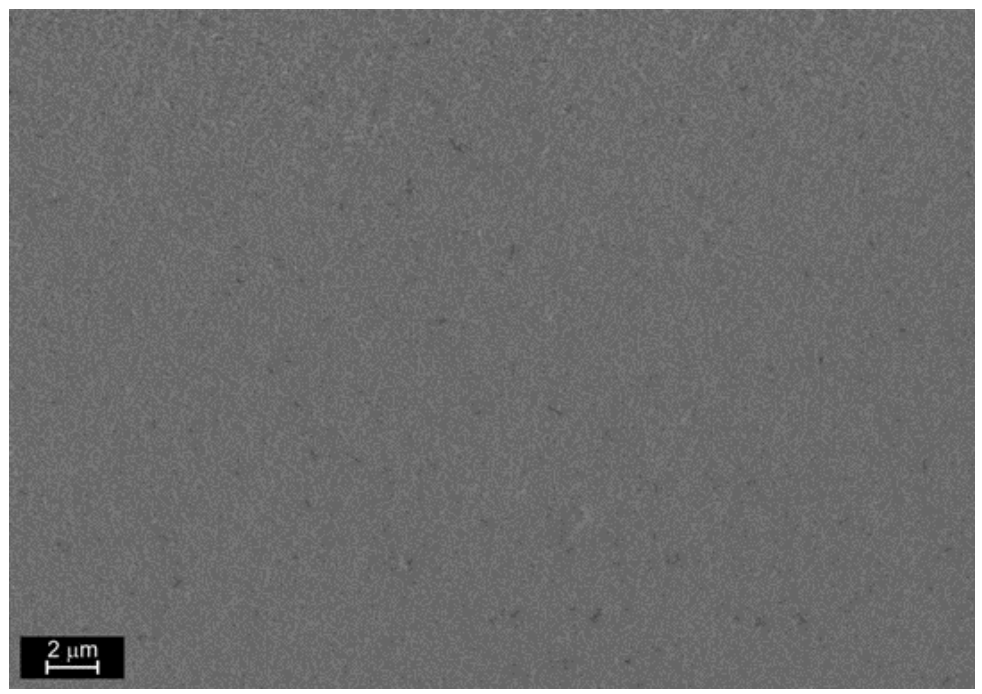

Figura 4: Micrografía SEM de la superficie de fractura de la película TPS-OQ-TPS. 
El análisis microestructural de la película evidenció una fractura vítrea como consecuencia de la inmersión del material en nitrógeno líquido. No se detectó la presencia de partículas de OQ esféricas dispersas en la matriz de TPS. Esto podría atribuirse a la buena disolución de los derivados en ácido acético antes de su incorporación en la película bicapa de TPS como así también a la eficiencia del proceso de termocompresión del material final. Además, la eficacia del procesamiento junto con la buena compatibilidad química entre los OQ y la matriz de TPS, se corroboraron debido a la ausencia de fases dispersas.

Dada la capacidad antimicrobiana de los OQ, el propósito de incorporar estos derivados a una matriz de almidón es desarrollar envases activos para alimentos. Así, la determinación de las propiedades ópticas resulta crucial ya que condicionan la aceptación visual del producto por parte del consumidor. Los parámetros de cromaticidad $a$ y $b$ de la película TPS-OQ-TPS resultaron ser de $-0,97 \pm 0,18$ y 6,20 $\pm 1,30$, respectivamente. El valor relativamente alto de $b$ denota la tonalidad amarillenta de la película que se corresponde con la observación visual del material. Este desarrollo de color podría atribuirse principalmente a la reacción de degradación no enzimática de Maillard que sufren los derivados de quitosano. Los productos de las reacciones de Maillard son compuestos de Amadori y polímeros insolubles, conocidos como melanoidinas que le confieren la coloración observada al material final [24-25]. El valor de $L$ del material desarrollado fue de $87,00 \pm 0,70$. Respecto a la opacidad de la película TPS-OQ-TPS, esta propiedad óptica resultó de 66,40 \pm 2,50 AU x nm.

La incorporación de antimicrobianos al material del envase supone que los agentes activos difundan de manera controlada desde la matriz hacia la superficie del alimento. Esta liberación está condicionada por distintos factores: la estructura y el tamaño del agente activo como así también la composición y morfología de la matriz. Si bien el gradiente de concentración es la fuerza impulsora de la migración, las condiciones del medio también influyen en la liberación, siendo el grado de acidez del alimento envasado uno de los factores más relevantes. Dentro de este contexto, se evaluó la liberación de los oligómeros desde la película hacia medios líquidos simulantes de alimentos ácidos a tres valores de $\mathrm{pH}$. Cabe destacar que los mismos fueron seleccionados en función de los grados de acidez característicos de una amplia variedad de alimentos.

La Tabla 2 incluye el porcentaje de difusión de los oligómeros desde la película TPS-OQ-TPS hacia medios líquidos a distintos $\mathrm{pH}$ en función del tiempo.

Tabla 2: Porcentaje de difusión de los oligómeros desde la película TPS-OQ-TPS a distintos tiempos hacia un medio líquido simulante con diferentes grados de acidez $(\mathrm{pH}=3,5, \mathrm{y} 7)$.

\begin{tabular}{|c|c|c|c|c|c|}
\hline \multirow{2}{*}{$\begin{array}{c}\text { pH DEL MEDIO } \\
\text { LÍQUIDO SIMULANTE }\end{array}$} & \multicolumn{5}{|c|}{ DIFUSIÓN DE LOS OLIGÓMEROS (\%) } \\
\hline & $t=30 \min$ & $t=60 \min$ & $t=120 \min$ & $t=150 \mathrm{~min}$ & $t=180 \min$ \\
\hline 3 & $67,19 \pm 2,05$ & $74,73 \pm 2,12$ & $75,52 \pm 2,85$ & $91,20 \pm 1,86$ & $89,82 \pm 0,89$ \\
\hline 5 & $64,54 \pm 2,39$ & $69,27 \pm 1,52$ & $75,25 \pm 0,93$ & $84,78 \pm 2,93$ & $89,89 \pm 1,34$ \\
\hline 7 & $58,18 \pm 0,20$ & $65,90 \pm 2,36$ & $66,95 \pm 2,57$ & $76,97 \pm 2,93$ & $85,86 \pm 4,16$ \\
\hline
\end{tabular}

En primer lugar, es relevante mencionar que los derivados estudiados fueron capaces de migrar desde la matriz de almidón hacia los tres medios ensayados. En todos los casos estudiados, la mayor velocidad de difusión tuvo lugar durante los primeros 30 min de inmersión. A partir de la primera hora, la concentración del derivado en dichos medios siguió incrementándose lentamente en el tiempo ensayado. Adicionalmente, bajos valores de $\mathrm{pH}$ favorecieron la difusión del oligosacárido hacia el medio líquido. Así, la difusión alcanzada al máximo tiempo ensayado resultó de $90 \%$ para los medios a pH 3, 5; mientras que para el pH 7 resultó ser de $86 \%$. Este resultado puede atribuirse a la mayor afinidad de estos derivados del quitosano con entornos de bajo $\mathrm{pH}$, ya que la protonación del grupo amino favorece su disolución y consecuentemente su difusión.

Teniendo en cuenta la capacidad que posee el quitosano y sus derivados de inhibir el crecimiento de hongos y levaduras, se seleccionó como alimento de referencia un producto fresco altamente susceptible al deterioro por contaminación con este tipo de microorganismos. Particularmente, las frutillas son frutas muy perecederas con una vida útil corta, de aproximadamente siete días si se conservan en condiciones adecuadas $\left(0^{\circ} \mathrm{C}\right.$ y alta humedad relativa). Además, poseen una epidermis muy fina que las hace sensibles a golpes y magulladuras y muy susceptibles al ataque de hongos y levaduras. Todos estos factores impulsaron el desarrollo de diferentes prácticas y metodologías que permitan prolongar la vida útil, garantizando la calidad nutricional, organoléptica y microbiológica post-cosecha de estas frutas. Para evaluar comparativamente la 
efectividad de los OQ en solución y contenidos en el material del envase, se realizaron estudios de capacidad antimicrobiana empleando frutillas frescas como alimento de referencia. La Figura 5 muestra el recuento de hongos y levaduras de las frutillas control $(C)$, las tratadas con la solución conteniendo los oligómeros $\left(O Q_{s o l}\right)$ mediante la técnica de spray, como así también las frutas envasadas en bolsas a base de TPS-OQ-TPS $\left(O Q_{\text {película }}\right)$.

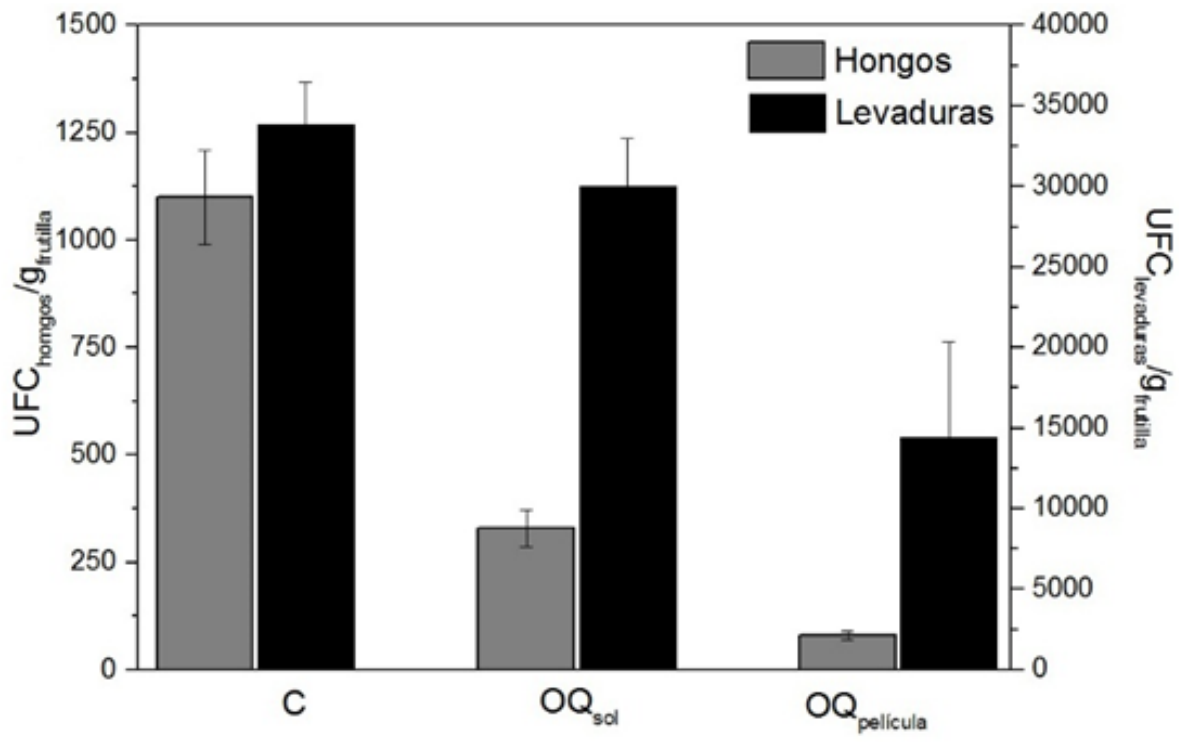

Figura 5: Recuento de hongos y levaduras de frutillas control $(C)$, tratadas con la solución conteniendo los oligómeros $\left(O Q_{s o l}\right)$ mediante la técnica de spray, como así también los frutos envasados en bolsas a base de TPS-OQ-TPS $\left(O Q_{p e l i c u l a}\right)$.

Como puede observarse en la Figura 5, la técnica de spray empleando la solución de OQ inhibió significativamente el desarrollo de hongos en las frutillas, alcanzándose una reducción del $70 \%$ en el crecimiento de estos microorganismos. En tanto que la incorporación de los derivados de quitosano en el material de envase de TPS-OQ-TPS potenció el efecto antifúngico de este agente activo, disminuyendo el desarrollo de hongos en los frutos envasados en un $76 \%$ respecto del spray. En relación al recuento de levaduras, no se observó una reducción notoria entre el control y el spray con la solución de OQ. Sin embargo, el uso de envases activos de TPS-OQ-TPS evidenció una mayor capacidad de inhibición del desarrollo de levaduras en los frutos estudiados, observándose una reducción del $52 \%$ del crecimiento de estos microorganismos.

Comparando la eficacia del agente activo aplicado por spray con la incorporación del mismo al material del envase, este estudio evidenció un incremento notorio de la protección del alimento contra el ataque de hongos y levaduras cuando los OQ se encuentran contenidos en la matriz de almidón. Estos resultados podrían atribuirse a que la liberación controlada de los derivados desde el envase hacia las frutillas garantiza un suministro gradual de los $\mathrm{OQ}$, incrementando así la vida útil del alimento. Esta migración paulatina de los OQ implica un mayor tiempo de permanencia de estos agentes activos sobre la superficie de las frutillas, donde su acción es requerida, minimizando la contaminación microbiana del producto. Por otra parte, los envases actúan como barrera física de protección de los frutos permitiendo además el intercambio gaseoso del producto con el medio.

\section{CONCLUSIONES}

En este trabajo se obtuvieron películas a base de almidón de maíz termoplástico conteniendo oligómeros de quitosano como agentes antimicrobianos. Estos materiales se emplearon para desarrollar envases tipo bolsa para productos frutihortícolas frescos tales como frutillas enteras. La capacidad antimicrobiana de estos envases se estudió comparativamente con el método convencional de spray para extender la vida útil de estos productos alimenticios. Los oligómeros considerados en este estudio fueron sintetizados previamente en el laboratorio a partir de la degradación oxidativa de quitosano asistida con radiación microondas, obteniéndose derivados de baja masa molar y grado de polimerización. En primera instancia, estos oligómeros fueron caracterizados morfológica y estructuralmente. El procesamiento de obtención de la película activa bicapa fue optimizado, obteniéndose un material homogéneo, translúcido y de coloración ámbar-amarillenta. El análisis 
de la microestructura de la película evidenció la compatibilidad entre la matriz de almidón termoplástico y los oligoquitosanos como así también la buena integridad del material final. Se determinaron las propiedades ópticas de la película activa a partir de la medición del color y opacidad de la misma. Se demostró que la difusión de los oligómeros desde la matriz polimérica hacia un medio líquido depende del grado de acidez del medio simulante, siendo este proceso favorecido por bajos valores de $\mathrm{pH}$. A partir de un estudio microbiológico, se demostró que el uso de envases activos con capacidad antimicrobiana resulta más eficiente que el método de aplicación por spray de una solución del agente activo sobre frutillas frescas.

\section{AGRADECIMIENTOS}

Los autores agradecen al Consejo Nacional de Investigaciones Científicas y Técnicas (CONICET) y a la Universidad Nacional del Sur (UNS).

\section{BIBLIOGRAFÍA}

[1] MA, X., CHANG, P.R., YU, J., et al., "Properties of biodegradable citric acid-modified granular starch/thermoplastic pea starch composites", Carbohydrate Polymers, v. 75, n. 1, pp. 1-8, Ene. 2009.

[2] LIU, H., ADHIKARI, R., GUO, Q., et al., "Preparation and characterization of glycerol plasticized (highamylose) starch-chitosan films", Journal of Food Engineering, v. 116, n. 2, pp. 588-597, May 2013.

[3] LOPEZ, O., GARCIA, M.A., VILLAR, M.A., et al., "Thermo-compression of biodegradable thermoplastic corn starch films containing chitin and chitosan", LWT - Food Science and Technology, vol. 57, no. 1, pp. 106-115, Jun. 2014.

[4] LONG, N.N. V., JOLY, C., DANTIGNY, P., "Active packaging with antifungal activities", International Journal of Food Microbiology, v. 220, pp. 73-90, Mar. 2016.

[5] AZARAKHSH, N., OSMAN, A., GHAZALI, H.M., et al., "Lemongrass essential oil incorporated into alginate-based edible coating for shelf-life extension and quality retention of fresh-cut pineapple", Postharvest Biology and Technology, vol. 88, pp. 1-7, Feb. 2014.

[6] OTONI, C.G., PONTES, S.F.O., MEDEIROS, E.A.A., et al., "Edible films from methylcellulose and nanoemulsions of love bud (Syzygium aromaticum) and oregano (Origanum vulgare) essential oils as shelf life extenders for sliced bread", Journal of Agricultural and Food Chemistry, v. 62, n. 22, pp. 5214-5219, Jun. 2014.

[7] KECHICHIAN, V., DITCHFIELD, C., VEIGA-SANTOS, P., et al., "Natural antimicrobial ingredients incorporated in biodegradable films based on cassava starch", LWT - Food Science and Technology, v. 43, n. 7, pp. 1088-1094, Sep. 2010.

[8] LÓPEZ, O.V., GARCÍA, M.A., ZARITZKY, N.E., "Film forming capacity of chemically modified corn starches", Carbohydrate Polymers, v. 73, n. 4, pp. 573-581, Sep. 2008.

[9] SHAO, J., YANG, Y., ZHONG, Q., "Studies on preparation of oligoglucosamine by oxidative degradation under microwave irradiation", Polymer Degradation and Stability, v. 82, n. 3, pp. 395-398, May. 2003.

[10] ZUÑIGA, A., DEBBAUDT, A., ALBERTENGO, L., et al., "Synthesis and characterization of n-propyln-methylene phosphonic chitosan derivative", Carbohydrate Polymers, v. 79, n. 2, pp. 475-480, Ene. 2010.

[11] CASTILLO, L., LÓPEZ, O., LÓPEZ, C., et al., "Thermoplastic starch films reinforced with talc nanoparticles", Carbohydrate Polymers, v. 95, n. 2, pp. 664-674, Jun. 2013.

[12] LÓPEZ, O.V., CASTILLO, L.A., GARCÍA, M.A., et al., "Food packaging bags based on thermoplastic corn starch reinforced with talc nanoparticles", Food Hydrocolloids, v. 43, pp. 18-24, Ene. 2015.

[13] ZHENG, X., YIN, Y., JIANG, W., et al., "Synthesis and characterization of low molecular weight chitosan", BioResources, v. 10, n. 2, pp. 2338-2349, Feb. 2015.

[14] KHANMOHAMMADI, M., ELMIZADEH, H., GHASEMI, K., "Investigation of size and morphology of chitosan nanoparticles used in drug delivery system employing chemometric technique", Iranian Journal of Pharmaceutical Research, v. 14, n. 3, pp. 665-675, 2015.

[15] COSTA-JÚNIOR, E. S., PEREIRA, M.M., MANSUR, H.S., "Properties and biocompatibility of chitosan films modified by blending with PVA and chemically crosslinked", Journal of Material Science: Materials in Medicine, v. 20, n. 2, pp. 553-561, Feb. 2009. 
[16] ZAINAL, Z., HUI, L.K., HUSSEIN, M.Z., et al., "Characterization of $\mathrm{TiO}_{2}$-chitosan/glass photocatalyst for the removal of a monoazo dye via photodegradation-adsorption process", Journal of Hazardous Materials, vol. 164, no. 1, pp. 138-145, May. 2009.

[17] AZIZ, M.S.A., NAGUIB, H.F., SAAD, G.R., "Nanocomposites based on chitosan-graft-poly(n-vinyl-2pyrrolidone): synthesis, characterization, and biological activity", International Journal of Polymeric Materials and Polymeric Biomaterials, v. 64, n. 11, pp. 578-586, Oct. 2015.

[18] ZAKARIA, Z., IZZAH, Z., JAWAID, M., et al., "Effect of degree of deacetylation of chitosan on thermal stability and compatibility of chitosan-polyamide blend", BioResources, v. 7, n. 4, pp. 5568-5580, Ago. 2012.

[19] NALLAMUTHU, I., DEVI, A., KHANUM, F., "Chlorogenic acid loaded chitosan nanoparticles with sustained release property, retained antioxidant activity and enhanced bioavailability", Asian Journal of Pharmaceutical Sciences, v. 10, n. 3, pp. 203-211, Jun. 2015.

[20] CORAZZARI, I., NISTICÒ, R., TURCI, F., et al., "Advanced physico-chemical characterization of chitosan by means of TGA coupled on-line with FTIR and GCMS: Thermal degradation and water adsorption capacity", Polymer Degradation and Stability, v. 112, pp. 1-9, Feb. 2015.

[21] MOUSSOUT, H., AHLAFI, H., AAZZA, M., et al., "Kinetics and mechanism of the thermal degradation of biopolymers chitin and chitosan using thermogravimetric analysis", Polymer Degradation and Stability, v. 130, pp. 1-9, Ago. 2016.

[22] ZIEGLER-BOROWSKA, M., CHEŁMINIAK, D., KACZMAREK, H. "Thermal stability of magnetic nanoparticles coated by blends of modified chitosan and poly(quaternary ammonium) salt", Journal of Thermal Analysis and Calorimetry, v. 119, n. 1, pp. 499-506, Ene. 2015.

[23] MARROQUIN, J.B., RHEE, K.Y., PARK, S.J. "Chitosan nanocomposite films: Enhanced electrical conductivity, thermal stability, and mechanical properties", Carbohydrate Polymers, v. 92, n. 2, pp. 1783 1791, Feb. 2013.

[24] LECETA, I., GUERRERO, P., DE LA CABA, K., "Functional properties of chitosan-based films", Carbohydrate Polymers, vol. 93, no. 1, pp. 339-346, Mar. 2013.

[25] YASIR, S.B.M., SUTTON, K.H., NEWBERRY, M.P., et al., "The impact of Maillard cross-linking on soy proteins and tofu texture", Food Chemistry, v. 104, n. 4, pp. 1502-1508, Feb. 2007. 